\title{
COMPLETE $Q$-MATRICES IN GENERAL ATTRIBUTE STRUCTURE MODELS
}

\author{
JÜRGEN HELLER \\ UNIVERSITY OF TÜBINGEN
}

July 23, 2019

Acknowledgements: I am grateful to Luca Stefanutti, Egidio Robusto, and Pasquale Anselmi for their valuable comments on previous versions of the paper.

Correspondence should be sent to

E-Mail: juergen.heller@uni-tuebingen.de

Phone: +497071 29-72979

Fax: +49 7071 29-3363

Address:

Department of Psychology

University of Tuebingen

Schleichstr. 4

72076 Tuebingen, Germany 
COMPLETE $Q$-MATRICES IN GENERAL ATTRIBUTE STRUCTURE MODELS

\begin{abstract}
In cognitive diagnostic assessment a property of the $Q$-matrix, usually referred to as completeness, warrants that the cognitive attributes underlying the observed behavior can be assessed uniquely. Characterizations of completeness were first derived under the assumption of independent attributes, and are currently under investigation for interdependent attributes. The dominant approach considers so-called attribute hierarchies, which are conceptualized through a partial order on the set of attributes. The present paper corrects and extends previously published results on this issue obtained for conjunctive attribute hierarchy models. Drawing upon results from knowledge structure theory it provides novel sufficient and necessary conditions for completeness of the $Q$-matrix, not only for conjunctive models on attribute hierarchies, but also on more general attribute structures.
\end{abstract}

Key words: attribute hierarchy models, identifiability, cognitive diagnostic models, conjunctive rule, knowledge structures. 


\section{Introduction}

Cognitive Diagnostic Models (CDMs; Bolt, 2007; de la Torre, 2009; Junker \& Sijtsma, 2001; K. K. Tatsuoka, 1990; C. Tatsuoka, 2002) were devised in order to assess the qualitative attributes characterizing an individual's ability to solve some items. To this end a whole range of different models was developed (e.g., see DiBello \& Stout, 2007; Rupp, Templin, \& Henson, 2010), most of them based on the latent class approach (Roussos, Templin, \& Henson, 2007). Attributes are conceived as properties of both persons and items. CDMs specify the relationship between items and attributes often in form of a binary matrix. The so-called $Q$-matrix (K. K. Tatsuoka, 1990) assigns to each item a specific subset of attributes, which are considered relevant to solving the item. The following refers to noncompensatory models implementing a conjunctive rule, where all attributes assigned to a problem are necessary for solving it.

In general, attributes may be considered as functioning independent from each other (K. K. Tatsuoka \& Boodoo, 2000), or they may be related in some way or the other. Characterizations of interesting properties of CDMs (like, e.g., identifiability) were obtained at first under the assumption of independent attributes. How these results change when attributes are interdependent is currently a highly active area (e.g., Cai, Tu, \& Ding, 2018; Köhn \& Chiu, 2018). This research strand is known under the label Attribute Hierarchy Models (AHMs). The dominant approach conceptualizes attribute hierarchies through a binary relation on the set of attributes.

CDMs are in close correspondence to models that were considered in Knowledge Structure Theory (KST; Doignon \& Falmagne, 1985). These two fields developed in parallel for several decades, with essentially no crosstalk between them. It was only recently that Heller, Stefanutti, Anselmi, and Robusto $(2015,2016)$ showed that a particular class of CDMs is equivalent to probabilistic KST models. To be more precise, they proved that the multi-strategy DINA model (MS-DINA; de la Torre \& Douglas, 2008) is equivalent to the so-called CBLIM (Heller et al., 2015), a competence-based extension of the basic local independence model (BLIM; Falmagne, 1989; Doignon \& Falmagne, 1999), which is the predominant probabilistic model in KST. This includes identifying popular CDMs as submodels of the CBLIM: the DINA model (E. H. Haertel, 1984, 1989, 1990) is equivalent to the conjunctive CBLIM, and the DINO model (Templin \& Henson, 2006) is equivalent to the disjunctive CBLIM.

In pointing out the intimate relation between the two fields, Heller et al. (2015) expressed the hope that this may contribute to facilitating the communication between the two camps, leading to more rapid advances in both research areas. This expectation might have been overly optimistic though, realizing the fundamental differences in the theoretical perspective and aims (due to the distinct origins in psychometrics and mathematical psychology), and maybe even more important - in the formalism and notation used. The present paper wants 


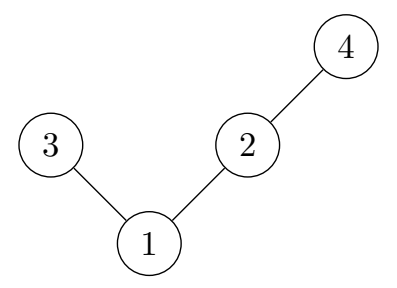

FiguRE 1.

Line diagram of a divergent attribute hierarchy. The convention is that line segments represent upward-directed arrows.

to take a further step in bridging the gap. It argues that KST can handle general kinds of attribute structures in a quite natural way. Exploiting this advantage, results that have been obtained in KST can be transferred to AHMs, thereby going beyond the current state. On the other hand, the $Q$-matrix design in CDM opens up a new perspective that leads to extending available KST results.

The paper is structured as follows. While Section 2 provides a gentle introduction into the basic notions defining AHMs, Section 3 points out their direct counterparts in a competence-based extension of KST (for an overview see Heller, Ünlü, \& Albert, 2013). Based on these correspondences, Section 4 shows that attribute hierarchies as considered by E. Haertel and Wiley (1994) and Leighton, Gierl, and Hunka (2004) may be taken into account in two different, but equivalent ways. Section 5 draws upon basic results from KST to transfer a characterization of more general attribute structures to CDM. Characterizations of the identifiability of AHMs as well as general attribute structure models are provided in Section 6. This generalizes a recently published result on the identifiability of AHMs by Cai et al. (2018), and corrects a false theorem by Köhn and Chiu (2018). While proofs are formulated within the KST framework, results are immediately recasted in CDM terms.

\section{Attribute Hierarchy Models}

For introducing AHMs, and for spelling out the correspondence to KST in the subsequent section, we refer to an example taken from Cai et al. (2018, Figure 2). For the terminology we refer to Leighton et al. (2004). In particular, the term attribute hierarchy is reserved for the situations considered there, where attributes are partially ordered. Cai et al. (2018) assume a so-called divergent attribute hierarchy on the attributes 1,2,3,4, which is illustrated in Figure 1.

The line diagram represents a relation that may exist between two attributes by a line segment, or a sequence of line segments, each of which is interpreted as an upward-directed 
arrow. The corresponding reachability matrix $\mathbf{R}$ reads

$$
\mathbf{R}=\left(\begin{array}{llll}
1 & 1 & 1 & 1 \\
0 & 1 & 0 & 1 \\
0 & 0 & 1 & 0 \\
0 & 0 & 0 & 1
\end{array}\right)
$$

The reduced $Q$-matrix is given by

$$
\mathbf{Q}_{r}=\left(\begin{array}{llllll}
1 & 1 & 1 & 1 & 1 & 1 \\
0 & 1 & 0 & 1 & 1 & 1 \\
0 & 0 & 1 & 1 & 0 & 1 \\
0 & 0 & 0 & 0 & 1 & 1
\end{array}\right) .
$$

Each of the columns of $\mathbf{Q}_{r}$ is a binary vector representing a potential item, for which the required attributes adhere to the assumed hierarchy. According to Leighton et al. (2004, p. 213) the reduced $Q$-matrix represents "the attribute blueprint or cognitive specifications for test construction". Notice that the columns in $\mathbf{Q}_{r}$ coincide with the permissible attribute patterns $\mathbf{M}$, except for a 0 -vector that is to be added, i.e.,

$$
\mathbf{M}=\left(\begin{array}{lllllll}
0 & 1 & 1 & 1 & 1 & 1 & 1 \\
0 & 0 & 1 & 0 & 1 & 1 & 1 \\
0 & 0 & 0 & 1 & 1 & 0 & 1 \\
0 & 0 & 0 & 0 & 0 & 1 & 1
\end{array}\right)
$$

Notice that by construction $\mathbf{M}$ always contains the 0 -vector and the 1-vector, and for the remainder we make the assumption that this also holds for any arbitrary matrix $\mathbf{M}$ of permissible attribute patterns that will be considered. Confining consideration to the permissible attribute patterns, the expected response patterns can be generated. The binary vector in each of the rows of the matrix

$$
\left(\begin{array}{llll}
0 & 0 & 0 & 0 \\
1 & 0 & 0 & 0 \\
1 & 1 & 0 & 0 \\
1 & 0 & 1 & 0 \\
1 & 1 & 1 & 0 \\
1 & 1 & 0 & 1 \\
1 & 1 & 1 & 1
\end{array}\right)
$$

represents the expected response pattern that results from the permissible attribute pattern in the respective column of $\mathbf{M}$ for the $Q$-matrix $\mathbf{Q}=\mathbf{R}$ as specified in (1).

Identifiability of the considered model is essential for cognitive diagnosis. With respect to the $Q$-matrix, identifiability refers to the important property that, given an expected response 
pattern, the underlying attribute pattern from which it was generated is uniquely determined. In CDM the term complete Q-matrix was coined by Chiu, Douglas, and Li (2009) to characterize $Q$-matrices that allow for this kind of unique attribute assessment. Assuming a conjunctive rule, identifiability in the case of independent attributes has been studied widely. It was shown that a $Q$-matrix is complete if and only if for each attribute it contains an item that requires this attribute only (e.g., Chiu et al., 2009, Lemma 1). In other words, a $Q$-matrix is complete if and only if it contains the $(k \times k)$ identity matrix as a submatrix, with $k$ the number of attributes. In recently published papers Cai et al. (2018) and Köhn and Chiu (2018) independently claim that they have generalized this result to attribute hierarchies (as suggested by Leighton et al. (2004)). The following formulation restates the claim within the terminology introduced above. Notice that Köhn and Chiu (2018) consider the DINA model and use a different, but in this context equivalent notion of completeness (see Appendix for details).

Claim. Under the regime of a conjunctive rule, for a given attribute hierarchy with reachability matrix $\mathbf{R}$ the $Q$-matrix is complete if and only if it contains $\mathbf{R}$ as a submatrix.

In Theorem 2 of Cai et al. (2018) this claim is formulated within the framework of a restricted $Q$-matrix design, assuming that the item-attribute assignments should reflect the specified attribute hierarchy (e.g., Leighton et al., 2004; C. Tatsuoka, 2009). So, an item requesting any attribute should also request all of its prerequisites, which confines consideration to $Q$-matrices that are sub-matrices of $\mathbf{Q}_{r}$. This is a clear limitation, and one would be interested in a characterization of the general case, which refrains from imposing such kind of constraints, and is sometimes referred to as independent $Q$-matrix design (de la Torre, Hong, \& Deng, 2010; Liu, Huggins-Manley, \& Bradshaw, 2017; Templin \& Bradshaw, 2014). This is exactly the situation considered in Theorem 1 of Köhn and Chiu (2018). However, we argue that the "only if" part of this theorem is invalid, and the "proof" provided by the authors is flawed. Probably the most easily traceable counterexample results from taking the identity matrix

$$
\mathbf{I}=\left(\begin{array}{llll}
1 & 0 & 0 & 0 \\
0 & 1 & 0 & 0 \\
0 & 0 & 1 & 0 \\
0 & 0 & 0 & 1
\end{array}\right)
$$

as the $Q$-matrix $\mathbf{Q}_{I}=\mathbf{I}$. The following observations are immediate. It is obvious that for $\mathbf{Q}=\mathbf{R}$ the reachability matrix is contained as a submatrix, while this is not the case for the identity matrix $\mathbf{Q}_{I}$. However, based on the matrix $\mathbf{M}$ of permissible attribute patterns in (3), both $Q$-matrices generate the very same matrix of expected response patterns as specified in (4) (which mathematically is nothing but the transpose of $\mathbf{M}$ ). This observation falsifies the assertion that containing the reachability matrix as a submatrix is a necessary condition for a 
$Q$-matrix to be complete.

The present paper investigates the issue. It not only provides a sufficient and necessary condition characterizing complete $Q$-matrices in attribute hierarchy models within an independent $Q$-matrix design, but also develops a characterization of completeness for more general types of attribute structures. This is achieved by exploiting the correspondences between CDM and KST which are outlined in the next section.

\section{CDM-KST Correspondences}

All of the notions introduced in the previous section have counterparts in KST (see Heller et al., 2015, for details). In contrast to the vector-based notation of CDM introduced above, KST is formulated within a set-theoretic framework. This has clear deficits from a computational point of view, but furthers a structural perspective, which is more likely to generate theoretical insights. Notice that Köhn and Chiu (2018) try to capitalize on this by pursuing a lattice-theoretic approach which essentially reframes well-known KST results without explicitly referring to them.

In KST attributes are simply called skills, and the set of skills is usually denoted by $S$, so that in our example $S=\{1,2,3,4\}$. The reachability matrix $\mathbf{R}$ then is the matrix representation of a binary relation $\leq$ on $S$, which is assumed to be a partial order (i.e., reflexive, transitive, and antisymmetric). The relation $\leq$ is called a precedence relation, and we have $s \leq t$ whenever skill $s \in S$ is a prerequisite to skill $t \in S$.

The reduced $Q$-matrix $\mathbf{Q}_{r}$ corresponds to a skill map $\tau: Q \rightarrow 2^{S} \backslash\{\emptyset\}$, a mapping from the set of items $Q$ (called knowledge domain in KST) into the collection of nonempty subsets of $S$. Because the columns of $\mathbf{Q}_{r}$ are interpreted as items, we may define the domain by $Q=\{a, b, c, d, e, f\}$, and the skill map by

$$
\tau(a)=\{1\}, \tau(b)=\{1,2\}, \tau(c)=\{1,3\}, \tau(d)=\{1,2,3\}, \tau(e)=\{1,2,4\}, \tau(f)=S .
$$

As with the columns of $\mathbf{Q}_{r}$, the subsets of skills assigned by $\tau$ (which are called competencies) receive a conjunctive interpretation, so $\tau$ is called a conjunctive skill map. Notice that in constructing the $Q$-matrix different items are assigned to different attribute pattern. This means that the corresponding skill map $\tau$ is injective, and this property will be assumed throughout the paper without explicit mention.

Let $\mathcal{C}$ denote the particular collection of subsets of $S$ that constitutes KST's counterpart of the matrix $\mathbf{M}$ of permissible attribute patterns. For the example this means that

$$
\mathcal{C}=\{\emptyset,\{1\},\{1,2\},\{1,3\},\{1,2,3\},\{1,2,4\}, S\} .
$$

The collection $\mathcal{C}$ - illustrated in Figure 2 - is called an ordinal competence space on $S$, as it is derived from the partial order $\leq$ on $S$ through

$$
C \in \mathcal{C} \text { iff }(s \leq t, t \in C \text { implies } s \in C)
$$




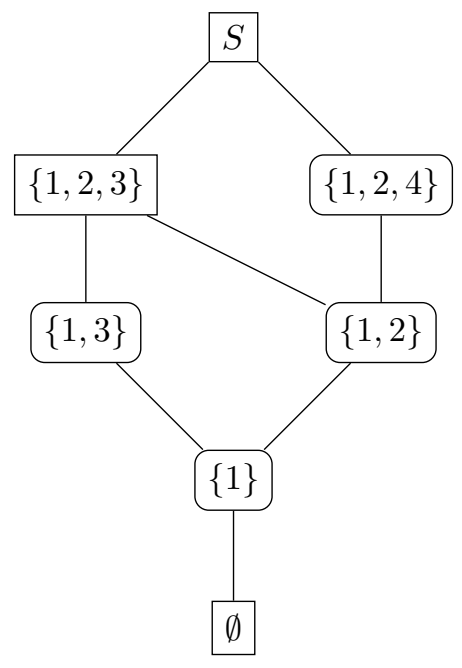

Figure 2.

Ordinal competence space corresponding to the attribute hierarchy in Figure 1.

for all $C \subseteq S$. The elements $C \in \mathcal{C}$ are called competence states. Notice that the ordinal competence space has the property, that, given two competence states in $\mathcal{C}$, also their union and their intersection is a competence state in $\mathcal{C}$. Moreover, $\mathcal{C}$ is discriminative, meaning that there are no distinct elements in $S$ that appear in exactly the same competence states. More formally, if there are $s, t \in S$ such that $s \in C$ if and only if $t \in C$ for all $C \in \mathcal{C}$, then $s=t$. These properties are not incidental. It was shown that there is a one-to-one correspondence between the partial orders $\leq$ on $S$ and the discriminative collections of subsets of $S$ containing at least $\emptyset$ and $S$ that are closed under union as well as intersection (Birkhoff, 1937).

A mapping $p: \mathcal{C} \rightarrow 2^{Q}$ represents the derivation of the expected response patterns from the permissible attribute patterns. The so-called problem function assigns to each competence state $C \in \mathcal{C}$ the subset of items

$$
p(C)=\{q \in Q: \tau(q) \subseteq C\}
$$

that can be solved within $C$, the delineated knowledge state. The collection $\mathcal{K}$ of delineated knowledge states, the delineated knowledge structure, is the range of $p$ (i.e., $p(\mathcal{C})=\mathcal{K}$ ). In general, a mapping $p: \mathcal{C} \rightarrow 2^{Q}$ is a problem function if it is monotonic with respect to set-inclusion, and satisfies $p(\emptyset)=\emptyset$ and $p(S)=Q$. For the ordinal competence space specified in (6) (see Figure 2), and the problem function $p$ associated to the skill map $\tau$ defined in (5) the delineated knowledge states are listed in Table 1.

It follows from Düntsch and Gediga (1995, Proposition 2.3) and Gediga and Düntsch (2002, Theorem 3.1) that there is a one-to-one correspondence between the considered conjunctive skill maps and particular types of problem functions. The relevant property characterizing these problem functions is that they preserve intersections (see also Heller \& 


\begin{tabular}{cc}
\hline$C$ & $p(C)$ \\
\hline$\emptyset$ & $\emptyset$ \\
$\{1\}$ & $\{a\}$ \\
$\{1,2\}$ & $\{a, b\}$ \\
$\{1,3\}$ & $\{a, c\}$ \\
$\{1,2,3\}$ & $\{a, b, c\}$ \\
$\{1,2,4\}$ & $\{a, b, d\}$ \\
$S$ & $Q$ \\
\hline
\end{tabular}

TABLE 1.

Competence states of the ordinal competence space specified in (6), and knowledge states delineated by the problem function $p$ associated to the skill map $\tau$ defined in (5).

Repitsch, 2008; Heller et al., 2013), i.e., if $\mathcal{C}$ is closed under intersection then also the delineated knowledge structure $\mathcal{K}$ is closed under intersection. This means that to each conjunctive skill map $\tau$ there is associated a uniquely determined intersection-preserving problem function $p$, and vice versa. For the considered example it is easily verified that the knowledge structure on $Q=\{a, b, c, d, e, f\}$ (see Table 1)

$$
\mathcal{K}=\{\emptyset,\{a\},\{a, b\},\{a, c\},\{a, b, c\},\{a, b, d\}, Q\}
$$

delineated by the conjunctive skill map $\tau$ defined in (5) is closed under intersection.

\begin{tabular}{ll}
\hline $\mathrm{CDM}$ & KST \\
\hline attribute & skill \\
$Q$-matrix & skill map \\
permissible attribute pattern & competence state \\
matrix of permissible attribute patterns & competence structure \\
expected response pattern & delineated knowledge state \\
matrix of expected response patterns & delineated knowledge structure \\
\hline
\end{tabular}

TABLE 2 .

Corresponding notions of CDM and KST.

Table 2 summarizes the correspondences that were exemplified above. It may serve as a dictionary for translating statements from KST to CDM, and vice versa.

\section{A Basic Equivalence}

This section introduces an approach which is pursued throughout the paper. Results are first derived within the KST framework, and then are translated into CDM terminology by 
means of the correspondences outlined above. Proceeding in this way, the development can benefit from a wealth of results available for the set-theoretic characterization of the relevant structures (not at all limited to KST). An alternative procedure would have to reinterpret the set-theoretic relations and operations (set-inclusion, union, and intersection) based on binary vectors within a lattice theoretic approach (e.g., Köhn \& Chiu, 2018), and to properly restate the set-theoretical results within this context. Although formally equivalent, the latter approach seems less naturally to us.

Let $Q$ be a nonempty finite set of dichotomous items. The knowledge state of a person is then defined as the collection $K \subseteq Q$ of all the items that this person is capable of solving. A knowledge structure $\mathcal{K}$ is a collection of knowledge states containing at least the empty set $\emptyset$ and $Q$ (Doignon \& Falmagne, 1985). The corresponding notions defined on a nonempty finite set $S$ of abstract skills, which are relevant to solving the items in $Q$, have already been introduced above. The competence state of a person is the subset $C \subseteq S$ of all the skills that this person has available. A competence structure $\mathcal{C}$ is a collection of competence states containing at least $\emptyset$ and $S$ (Korossy, 1997, 1999). As also mentioned above, the observable behavior is related to the underlying skills by means of a skill map $\tau: Q \rightarrow 2^{S} \backslash\{\emptyset\}$, assigning a nonempty subset of $S$ to each item $q \in Q$. The problem function $p: \mathcal{C} \rightarrow 2^{S}$ associated to $\tau$ is defined by (7). In the sequel, $\tau$ is assumed to be a conjunctive skill map, meaning that the skills in $\tau(q)$ are both necessary and sufficient for solving item $q$.

Collecting the relevant notions in a basic structure, the quadruple $(Q, S, \mathcal{C}, \tau)$ is introduced to denote a so-called conjunctive competence model (Heller, Anselmi, Stefanutti, \& Robusto, 2017). For the rest of this section let us assume that $\mathcal{C}$ is an ordinal competence space, which is in one-to-one correspondence to a partial order $\leq$ on $S$. By this we cover all the attribute hierarchies considered by Leighton et al. (2004).

The proof of Proposition 1 below will draw upon the following notions.

First, consider the mapping $\downarrow: 2^{S} \rightarrow \mathcal{C}$ that associates the downset

$$
\downarrow T=\{s \in S: s \leq t \text { for some } t \in T\}
$$

to any subset $T \subseteq S$. Forming the downset $\downarrow T$ means to include with each skill $t \in T$ all of its prerequisites $s \in S$ with $s \leq t$. It is easily seen that, formally, the mapping $\downarrow$ establishes a closure operator on the partial order $(S, \subseteq)$. This means that for all $U, V \subseteq S$ the following properties hold: (i) $U \subseteq \downarrow U$, (ii) $U \subseteq V$ implies $\downarrow U \subseteq \downarrow V$, (iii) $\downarrow$ ( $\downarrow U)=\downarrow U$. The competence states in the ordinal competence space $\mathcal{C}$ corresponding to the precedence relation $\leq$ are the closed elements, i.e., for all $T \subseteq S$ we have

$$
\downarrow T=T \text { iff } T \in \mathcal{C} .
$$

Second, define a mapping $c: 2^{S} \rightarrow \mathcal{C}$ by

$$
T \mapsto T^{c}=\bigcup\{U \in \mathcal{C}: U \subseteq T\}
$$


for all $T \subseteq S$. Notice that $T^{c}$ is a competence state of $\mathcal{C}$ because the latter is closed for union. It is routine to show that $T \mapsto T^{c}$ forms an interior operator on the partial order $(S, \subseteq)$. This means that for all $U, V \subseteq S$ the following properties hold: (i) $U^{c} \subseteq U$, (ii) $U \subseteq V$ implies $U^{c} \subseteq V^{c}$, (iii) $\left(U^{c}\right)^{c}=U^{c}$.

Now, given the arbitrary conjunctive skill map $\tau$, in the spirit of the restricted $Q$-matrix design construct a conjunctive skill map $\tau^{*}$ by

$$
\tau^{*}(q)=\downarrow \tau(q)
$$

for all $q \in Q$. Notice that the $\tau^{*}(q), q \in Q$, all are competence states in $\mathcal{C}$ due to (9). Let $p^{*}: 2^{S} \rightarrow 2^{Q}$ be the problem function associated to $\tau^{*}$, with its range denoted by $\mathcal{K}^{*}$. Notice that $p^{*}$ is defined on the whole powerset of $S$, meaning that we ignore existing dependencies between the skills at the level of the considered competence structure (which might be denoted by $\mathcal{C}^{*}=2^{S}$ ).

Proposition 1 claims that, within the present setting, interdependencies between skills can be accounted for in two different, but equivalent ways: either through a competence structure, or by a skill map, both respecting these dependencies.

Proposition 1. Let $(Q, S, \mathcal{C}, \tau)$ be a conjunctive competence model, with $\mathcal{C}$ an ordinal competence space, and let $\mathcal{K}$ denote the delineated knowledge structure. Moreover, let $\left(Q, S, \mathcal{C}^{*}=2^{S}, \tau^{*}\right)$ be a conjunctive competence model with $\tau^{*}$ defined on $\tau$ through (10), and let $\mathcal{K}^{*}$ denote the respective delineated knowledge structure. Then $\mathcal{K}=\mathcal{K}^{*}$.

Proof. For proving the equation $\mathcal{K}^{*}=\mathcal{K}$ we have to show that $p^{*}(T)=p\left(T^{c}\right)$ holds for all $T \subseteq S$. So, let $C \in \mathcal{C}$. Then

$$
p(C)=\{q \in Q: \tau(q) \subseteq C\} \subseteq\{q \in Q: \downarrow \tau(q) \subseteq \downarrow C\}=\left\{q \in Q: \tau^{*}(q) \subseteq C\right\}=p^{*}(C),
$$

where the inclusion follows by property (ii) of the closure operator $\downarrow$, and the subsequent equation by (9). Conversely, due to $\tau(q) \subseteq \tau^{*}(q)$, we have

$$
p^{*}(C)=\left\{q \in Q: \tau^{*}(q) \subseteq C\right\} \subseteq\{q \in Q: \tau(q) \subseteq C\}=p(C) .
$$

This provides $p(C)=p^{*}(C)$ for all $C \in \mathcal{C}$, which means that $\mathcal{K} \subseteq \mathcal{K}^{*}$.

Now, let $T \in 2^{S}$. Since $\tau^{*}(q) \in \mathcal{C}$ for all $q \in Q$, and because of the definition of $T^{c}$, it follows that $\tau^{*}(q) \subseteq T$ implies $\tau^{*}(q) \subseteq T^{c}$. As the converse implication is immediate from $T^{c} \subseteq T$, we have

$$
p^{*}(T)=\left\{q \in Q: \tau^{*}(q) \subseteq T\right\}=\left\{q \in Q: \tau^{*}(q) \subseteq T^{c}\right\}=p^{*}\left(T^{c}\right)
$$

Noticing that $T^{c} \in \mathcal{C}$, this finally provides $p^{*}(T)=p^{*}\left(T^{c}\right)=p\left(T^{c}\right)$ for all $T \subseteq S$, which completes the proof of $\mathcal{K}=\mathcal{K}^{*}$. 
Proposition 1 shows that there are different, but equivalent options for taking heed of dependencies between skills that are characterized by a partial order. Given a particular conjunctive skill map $\tau$ they may be accounted for by limiting consideration to the corresponding ordinal competence space $\mathcal{C}$, or by extending the skill map as in (10) and consider all potential competencies in $2^{S}$. These two accounts are equivalent in the sense that they cannot be distinguished empirically, because they predict exactly the same observable behavior.

Notice that, due to the first part of the proof of Proposition 1, we have the following corollary.

Corollary 1. Under the conditions of Proposition 1, let $p: \mathcal{C} \rightarrow 2^{Q}$ and $p^{*}: 2^{S} \rightarrow 2^{Q}$ be the problem functions associated to $\tau$ and $\tau^{*}$. Then $p$ and $p^{*}$ coincide on $\mathcal{C}$, i.e., $p=p^{*} \mid \mathcal{C}$.

In order to translate these results to $Q$-matrix design, let us take up again the counterexample from Section 2, and let $\tau$ correspond to the $k \times k$ identity matrix $\mathbf{I}$ on the set of $k$ attributes. Then $\tau^{*}$ defined on $\tau$ through (10) corresponds to the reachability matrix $\mathbf{R}$ of the given attribute hierarchy, i.e., $\mathbf{I}^{*}=\mathbf{R}$. In this equation the construction of forming downsets of competencies is applied to $Q$-matrices, which includes the following steps: for any $Q$-matrix $\mathbf{Q}$ consider the corresponding conjunctive skill map $\tau$, construct $\tau^{*}$ by forming downsets, and finally consider the $Q$-matrix $\mathbf{Q}^{*}$ corresponding to the latter (maybe keeping only a single one of multiple identical columns for parsimony reasons).

Then, Proposition 1 implies that exactly the same expected response patterns are obtained in two different ways. The following corollary provides the details.

Corollary 2. Let there be $k$ binary attributes, and let $\mathbf{M}$ be the matrix of permissible attribute patterns that respects an attribute hierarchy with reachability matrix $\mathbf{R}$. Then, under a conjunctive rule, the following procedures generate exactly the same expected response patterns:

(i) let the $Q$-matrix be the $k \times k$ identity matrix and confine consideration to the permissible attribute patterns in $\mathbf{M}$;

(ii) identify the $Q$-matrix with $\mathbf{R}$ and allow for all $2^{k}$ potential attribute patterns.

In the latter case predictions stay the same if expected response patterns are generated through the $Q$-matrix $\mathbf{R}$ from the matrix $\mathbf{M}$ of permissible attribute patterns only.

\section{General Attribute Structures}

The attribute hierarchies listed by Leighton et al. (2004) all form partial orders on a set of skills (or, equivalently, attributes) $S$. It was already mentioned above that the partial 


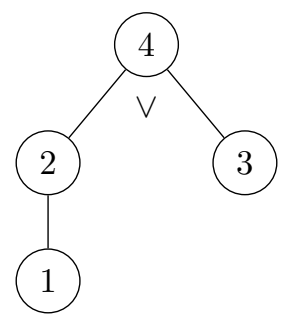

FiguRE 3.

Example of an attribute structure represented by an AND-OR graph.

orders on a set are in one-to-one correspondence to discriminative collections of subsets of $S$ containing at least $\emptyset$ and $S$, which are closed under both union and intersection (Birkhoff, 1937). Application of this result was outlined in the previous section. Doignon and Falmagne (1985) also provided a characterization of even more general orders, which may be represented by AND-OR graphs as illustrated in Figure 3. The key feature here is, that the set of prerequisites to a skill need not be unique. This reflects the fact that different sets of skills may furnish a person with all that is needed to acquire another skill (representing different learning paths). Figure 3 illustrates a situation where, from knowing that an individual has available skill 4, we may conclude that the individual has available either skill 2 (and thus its prerequisite 1), or alternatively skill 3. Doignon and Falmagne (1985) characterized the structure underlying AND-OR graphs by surmise systems, which assign subsets of skills (the alternative sets of prerequisites) to each skill. The surmise system $\sigma$ for the AND-OR graph in Figure 3 then reads

$$
\sigma(1)=\{\{1\}\}, \sigma(2)=\{\{1,2\}\}, \sigma(3)=\{\{3\}\}, \sigma(4)=\{\{1,2,4\},\{3,4\}\} .
$$

Doignon and Falmagne (1985) proved that surmise systems on $S$ are in one-to-one correspondence to collections of subsets of $S$ containing at least $\emptyset$ and $S$, which are closed under union. These collections of subsets are called competence spaces (Korossy, 1997, 1999). Figure 4 illustrates the competence space corresponding to the AND-OR graph in Figure 3.

The above arguments may be taken even one step further. Any arbitrary competence structure, which is a collection of subsets of $S$ containing at least $\emptyset$ and $S$ itself (not necessarily closed under union or intersection), may be considered a representation of dependencies between skills. A competence structure characterizes the case of independent skills if and only if it actually coincides with the powerset of $S$. This provides us with the tools for representing highly general types of attribute structures, which cannot be fully described by means of pairwise prerequisite relations between attributes.

The notion of an atom of a competence structure $\mathcal{C}$ on $S$ is at the core of this generalization. A subset $A \in \mathcal{C}$ is an atom at $s \in S$ if it is a minimal element (with respect to set-inclusion) in $\mathcal{C}$ containing $s$. It is called an atom if it is an atom at $s$ for some $s \in S$ (cf. Doignon \& Falmagne, 1999; Falmagne \& Doignon, 2011). The matrix, which results from 


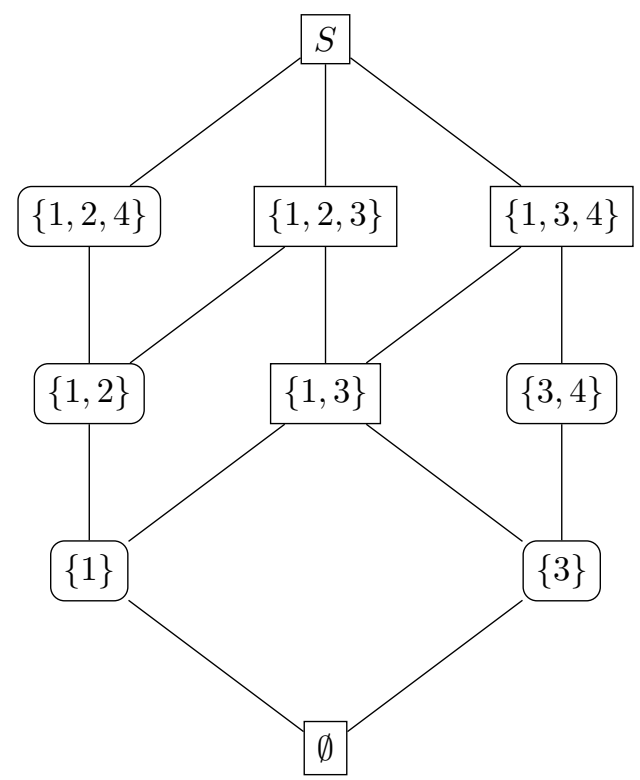

Figure 4.

Competence space corresponding to the attribute structure in Figure 3.

selecting the atoms of a given competence structure (properly translated into binary vectors) as its columns, will be called the matrix of the basic attribute patterns, and will be denoted by B.

The atoms in the competence structures illustrated in Figures 2 and 4 are marked by frames with rounded corners. It is easily checked that for the ordinal competence space in Figure 2 we have $\mathbf{B}=\mathbf{R}$, where $\mathbf{R}$ is the reachability matrix in (1). In exactly the same way the matrix

$$
\mathbf{B}=\left(\begin{array}{lllll}
1 & 0 & 1 & 0 & 1 \\
0 & 0 & 1 & 0 & 1 \\
0 & 1 & 0 & 1 & 0 \\
0 & 0 & 0 & 1 & 1
\end{array}\right)
$$

is obtained for the competence space depicted in Figure 4.

Notice that in a competence space $\mathcal{C}$ every competence state is equal to the union of all the atoms of $\mathcal{C}$ it contains. The matrix $\mathbf{B}$ of the basic attribute patterns, however, can be determined for any arbitrary competence structure $\mathcal{C}$, that is for any arbitrary matrix $\mathbf{M}$ of permissible attribute pattern (remember that by assumption $\mathbf{M}$ contains at least the 0 -vector and the 1-vector). The matrix $\mathbf{B}$ of the basic attribute patterns coincides with the reachability matrix $\mathbf{R}$ for attribute hierarchies, and it takes over the role of $\mathbf{R}$ for the more general types of attribute structures. 


\section{Complete $Q$-Matrices}

Heller et al. (2015) showed that the DINA model is equivalent to a conjunctive CBLIM. Based on this direct link between CDM and KST, the authors were able to draw upon results on the identifiability of the latter to characterize the identifiability of the DINA model. Being based on the structure of a conjunctive competence model, these results do not only hold for independent attributes, but cover the general attribute structures as introduced in Section 5 . By spelling out the implications in terms of properties of the $Q$-matrix, usually referred to as completeness (Chiu et al., 2009), Section 6 corrects an invalid claim by Köhn and Chiu (2018) and generalizes recently published results by Cai et al. (2018).

The following argues that the cited assertions about completeness of the $Q$-matrix for independent attributes and attribute hierarchies are special cases of results obtained within the KST framework. Translating these results into CDM terminology goes beyond the current state in this area of research. The CDM perspective targeting $Q$-matrix design, on the other hand, leads to generalizations of available KST results.

We first state the results of Heller et al. $(2015,2016)$ in terms of KST, with slight adaptations to the conventions used in the present paper. For this, let $\mathcal{C}$ be an arbitrary competence structure on a nonempty finite set $S$ of skills, and let $\tau: Q \rightarrow 2^{S} \backslash\{\emptyset\}$ denote a conjunctive skill map defined on a nonempty finite set of items $Q$. Then the conjunctive competence model $(Q, S, \mathcal{C}, \tau)$ is identifiable whenever its associated problem function $p: \mathcal{C} \rightarrow 2^{Q}$ is injective. The following notion was introduced by Heller et al. (2015): the skill map $\tau$ is said to respect the witness condition with respect to the competence structure $\mathcal{C}$ whenever

for every atom $A \in \mathcal{C}$ there is an item $q \in Q$ such that $\tau(q)=A$.

Heller et al. (2016, Proposition 9) prove that if $\tau$ respects the witness condition (W-C) then the associated problem function is injective. That is, the witness condition is a sufficient, but in general not a necessary condition for identifiability. An exception, which is explicitly mentioned in the cited proposition, is the case when $\mathcal{C}$ coincides with the powerset $2^{S}$. The witness condition $\left(\mathrm{W}-2^{S}\right)$ is sufficient and necessary for identifiability. As the atoms of the powerset $2^{S}$ are the singleton sets $\{s\}$ for $s \in S,\left(\mathrm{~W}-2^{S}\right)$ means that for every skill $s \in S$ there is an item $q \in Q$ such that $q$ requires exactly skill $s$. The translation of these results into CDM terms is formulated as a corollary to Proposition 9 of Heller et al. (2016).

Corollary 3. Let $\mathbf{M}$ be a matrix of permissible attribute pattern. Then, under a conjunctive rule, the $Q$-matrix is complete if it contains the matrix $\mathbf{B}$ of the basic attribute patterns for $\mathbf{M}$ as a submatrix. Given $\mathbf{M}$ is the incidence matrix (containing all potential attribute patterns), the $Q$-matrix is complete if and only if it contains the identity matrix (which in this case is the matrix of the basic attribute patterns) as a submatrix. 
The equivalence in the second sentence of Corollary 3 parallels Lemma 1 of Chiu et al. (2009). Notice that the equivalence can even be generalized to matrices $\mathbf{M}$ for which the matrix $\mathbf{B}$ of basic attribute patterns is the identity matrix (Heller et al., 2017, Proposition 1). However, already for attribute hierarchies the "only if" part does not hold in general, as stated in the first sentence of Corollary 3. This is at odds to Theorem 1 of Köhn and Chiu (2018), and a counterexample to the equivalence stated there is readily available. The example presented in Section 4 demonstrated that, given consideration is confined to the permissible attribute patterns, there are different $Q$-matrices that delineate the same expected response patterns. In particular, this was shown for selecting the identity matrix I vs. the reachability matrix $\mathbf{R}$ as the $Q$-matrix (because $\mathbf{I}^{*}=\mathbf{R}$ as a consequence of Corollary 1 ). Obviously, of these two $Q$-matrices only $\mathbf{R}$ satisfies the completeness criterion of Cai et al. (2018) requiring that the reachability matrix is to be contained as a submatrix. The necessity of this condition that the authors prove in their Theorem 2 is due to the constraints imposed by the restricted $Q$-matrix design they follow. It forces the construction to result in a submatrix of the reduced $Q$-matrix, which excludes I. The example shows that when this limitation is abolished, the criterion is no longer necessary, and the "only if" part of Theorem 1 of Köhn and Chiu (2018) is falsified.

As an example for more general attribute structures consider the AND-OR graph in Figure 3, which is captured by the competence space $\mathcal{C}$ illustrated in Figure 4 . The corresponding matrix of permissible attribute patterns reads

$$
\mathbf{M}=\left(\begin{array}{llllllllll}
0 & 1 & 0 & 1 & 1 & 0 & 1 & 1 & 1 & 1 \\
0 & 0 & 0 & 1 & 0 & 0 & 1 & 1 & 0 & 1 \\
0 & 0 & 1 & 0 & 1 & 1 & 1 & 0 & 1 & 1 \\
0 & 0 & 0 & 0 & 0 & 1 & 0 & 1 & 1 & 1
\end{array}\right)
$$

and its matrix $\mathbf{B}$ of basic attribute patterns is given by (11). Then the $Q$-matrix

$$
\mathbf{Q}=\left(\begin{array}{lllllll}
1 & 1 & 0 & 1 & 0 & 0 & 0 \\
0 & 1 & 0 & 1 & 0 & 1 & 0 \\
0 & 0 & 1 & 0 & 1 & 0 & 0 \\
0 & 0 & 0 & 1 & 1 & 0 & 1
\end{array}\right)
$$

contains $\mathbf{B}$ as the submatrix spanning the first five columns. Notice that the last two columns refer to items with non-permissible attribute patterns. The $Q$-matrix $\mathbf{Q}$ satisfies the sufficient condition for completeness formulated in Corollary 3. Figure 5 plots the expected response patterns as states of the corresponding delineated knowledge structure $\mathcal{K}$ on $Q=\{a, b, c, d, e, f, g\}$, where the item names $a$ to $g$ label the columns of $\mathbf{Q}$ from left to right. Injectiveness of the problem function is evident from comparing Figures 4 and 5 , because the two structures $(\mathcal{C}, \subseteq)$ and $(\mathcal{K}, \subseteq)$ are isomorphic.

The results obtained in Corollary 1 suggest to generalize the witness condition in case of partially ordered skills. Given a conjunctive competence model $(Q, S, \mathcal{C}, \tau)$, with $\mathcal{C}$ an ordinal 


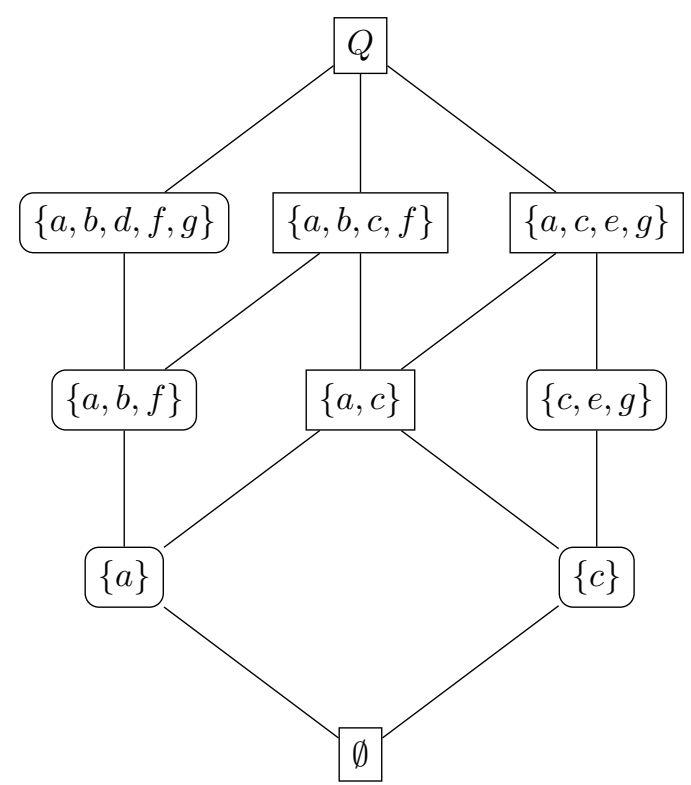

FiguRe 5.

Knowledge structure $\mathcal{K}$ delineated by the skill map corresponding to the $Q$-matrix in (12) based on the competence space $\mathcal{C}$ in Figure 4.

competence space, the skill map $\tau$ is said to respect the starred witness condition with respect to the competence structure $\mathcal{C}$ whenever

for every atom $A \in \mathcal{C}$ there is an item $q \in Q$ such that $\tau^{*}(q)=A$.

Notice that $\left(\mathrm{W}^{*}-\mathcal{C}\right)$ is weaker than $(\mathrm{W}-\mathcal{C})$. Returning to the example already discussed above, let $\tau$ correspond to the $k \times k$ identity matrix $\mathbf{I}$ on the set of $k$ attributes. So, the set of items $Q$ is such that for each $s \in S$ there is a $q \in Q$ with $\tau(q)=\{s\}$, and all $\tau(q)$ are of this form. Let $\mathcal{C}$ be an ordinal competence space that does not contain all of the singleton sets $\{s\}$, $s \in S$. Then $\tau$ does not respect the witness condition $(\mathrm{W}-\mathcal{C})$, but it respects the starred witness condition $\left(\mathrm{W}^{*}-\mathcal{C}\right)$.

The following proposition shows that the starred witness condition is both sufficient and necessary for injectiveness of the problem function in case of partially ordered skills.

Proposition 2. Let $(Q, S, \mathcal{C}, \tau)$ be a conjunctive competence model, with $\mathcal{C}$ an ordinal competence space, and let $p$ be the problem function associated to $\tau$. Then $\tau$ respects the starred witness condition $\left(\mathrm{W}^{*}-\mathcal{C}\right)$ if and only if $p$ is injective on $\mathcal{C}$.

Proof. The implication from left to right follows from Proposition 9 of Heller et al. (2016) in conjunction with Corollary 1. For the implication from right to left, assume that $\tau$ does not respect $\left(\mathrm{W}^{*}-\mathcal{C}\right)$, i.e., there is an atom $A$ at some $s \in S$ in $\mathcal{C}$ such that $\tau^{*}(q) \neq A$ for all $q \in Q$. 
Consider $B=\bigcup\left\{\tau^{*}(q): \tau^{*}(q) \subseteq A, q \in Q\right\}$. Then $B \in \mathcal{C}$, because for all $q \in Q$ the $\tau^{*}(q)$ are in $\mathcal{C}$, which in turn is closed under union. Moreover, it follows that $s \notin B$. Otherwise, there is a $r \in Q$ such that $s \in \tau^{*}(r) \subseteq A$. However, $\tau^{*}(r)$ is in $\mathcal{C}$ and by assumption it is distinct from $A$. Because $A$ is the unique atom at $s$, this implies the proper inclusion $A \subset \tau^{*}(r)$, a contradiction. So, $A \neq B$. But $p^{*}(A)=p^{*}(B)$, because $\tau^{*}(q) \subseteq A$ if and only if $\tau^{*}(q) \subseteq B$ for all $q \in Q$. Since $p$ coincides with $p^{*}$ on $\mathcal{C}$ by Corollary 1 , this means that $p$ is not injective.

This leads to the following generalization of the first assertion in Corollary 3 , which is to replace the flawed theorem by Köhn and Chiu (2018).

Corollary 4. Let $\mathbf{M}$ be the matrix of permissible attribute patterns that respects an attribute hierarchy with reachability matrix $\mathbf{R}$. Then, under a conjunctive rule, the $Q$-matrix $\mathbf{Q}$ is complete if and only if $\mathbf{Q}^{*}$ contains $\mathbf{R}$ as a submatrix.

It has to be emphasized that the proof of Proposition 2 draws heavily upon the assumption that $\mathcal{C}$ is closed under both union and intersection. So, the result will not generalize to arbitrary competence structures not satisfying these properties. Indeed, in general the witness condition is sufficient, but not necessary for $p$ being injective (Heller et al., 2016). But consider the following attenuated condition. Let $(Q, S, \mathcal{C}, \tau)$ be a conjunctive competence model. Then $\tau$ is said to respect the weak witness condition with respect to the competence structure $\mathcal{C}$ whenever

for every atom $A \in \mathcal{C}$ there is an item $q \in Q$ such that $\tau(q) \subseteq A$.

$(\mathrm{wW}-\mathcal{C})$

The weak witness condition formulates a 'conditio sine qua non', i.e., if the problem function associated to $\tau$ is injective then $\tau$ need to respect (wW-C) (Heller et al., 2016, Proposition 9). In order to translate this condition into CDM terms, let the binary relation $\mathbf{x} \sqsubseteq \mathbf{y}$ hold for binary vectors $\mathbf{x}, \mathbf{y}$ whenever $x_{i} \leq y_{i}$ holds for each of their components $x_{i}, y_{i}$ (appropriately indexed by $i$ ). The relation $\sqsubseteq$ forms the analog of set inclusion for binary vectors. Corollary 5 allows for identifying $Q$-matrices that are not complete.

Corollary 5. Let $\mathbf{M}$ be an arbitrary matrix of permissible attribute patterns. Then, under a conjunctive rule, if the $Q$-matrix $\mathbf{Q}$ is complete then for each column $\mathbf{b}_{k}$ of the matrix $\mathbf{B}$ of the basic attribute patterns for $\mathbf{M}$ there is a column $\mathbf{q}_{j}$ of $\mathbf{Q}$ such that $\mathbf{q}_{j} \sqsubseteq \mathbf{b}_{k}$.

Condition (wW-C) indeed formulates a weak constraint. Let $q \in Q$ be such that $\tau(q) \subseteq A$ for an atom $A \in \mathcal{C}$ at $s \in S$. Then (wW-C) does not require that $s$ is in $\tau(q)$. This suggests the following stronger version of the weak witness condition. Let $(Q, S, \mathcal{C}, \tau)$ be a conjunctive competence model. Then $\tau$ is said to respect the strict weak witness condition with respect to the competence structure $\mathcal{C}$ whenever

for every atom $A \in \mathcal{C}$ at some $s \in S$ there is an item $q \in Q$ such that $s \in \tau(q) \subseteq A$. (swW-C) 
This condition has not been considered before. The following shows that in case of partially ordered skills, with $\mathcal{C}$ being an ordinal competence space, (swW-C) is equivalent to the starred witness condition $\left(\mathrm{W}^{*}-\mathcal{C}\right)$.

Proposition 3. Let $(Q, S, \mathcal{C}, \tau)$ be a conjunctive competence model, with $\mathcal{C}$ an ordinal competence space. Then $\tau$ respects the strict weak witness condition (swW-C) if and only if it respects the starred witness condition $\left(\mathrm{W}^{*}-\mathcal{C}\right)$.

Proof. Assume that (swW-C) holds. Let $A \in \mathcal{C}$ be an atom at $s \in S$, and let $q \in Q$ be such that $s \in \tau(q) \subseteq A$. Then $\tau^{*}(q)=A$ because $\tau^{*}(q) \subseteq A$ by property (ii) of the downset closure operator, and $A \subseteq \tau^{*}(q)$ since $A$ is an atom at $s$. Thus, $\tau$ respects ( ${ }^{*}-\mathcal{C}$ ). Conversely, if $\tau$ respects $\left(\mathrm{W}^{*}-\mathcal{C}\right)$ and $A \in \mathcal{C}$ is an atom at $s \in S$, then there is a $q \in Q$ such that $\tau^{*}(q)=A$. This implies that $\tau(q) \subseteq A$. Moreover, we have $s \in \tau(q)$, because otherwise there is a $t \in \tau(q)$ distinct from $s$ such that $s \leq t$, and $t \not \leq s$ since $\leq$ is a partial order. This implies that $t \notin \downarrow\{s\} \in \mathcal{C}$. But $\downarrow\{s\} \subset A$ contradicts the assumption that $A$ is an atom at $s$. Thus, $($ swW-C) holds.

Translating Proposition 3 into CDM terms allows for dispensing with application of the *-operator on which Corollary 4 relies.

Corollary 6. Let $\mathbf{M}$ be the matrix of permissible attribute patterns that respects an attribute hierarchy with reachability matrix $\mathbf{R}$. Then, under a conjunctive rule, the $Q$-matrix $\mathbf{Q}$ is complete if and only if for all attributes $a_{i}$ and the column $\mathbf{r}_{k}$ of $\mathbf{R}$ that is minimal (with respect to $\sqsubseteq)$ among those satisfying $r_{i k}=1$, there is a column $\mathbf{q}_{j}$ of $\mathbf{Q}$ such that $q_{i j}=1$ and $\mathbf{q}_{j} \sqsubseteq \mathbf{r}_{k}$.

Considering the general case again, Heller et al. (2017) identified a condition, called the identifiability condition (IC), which is both sufficient and necessary for identifiability given any arbitrary competence structure and any conjunctive skill map.

Proposition 4. (Heller et al., 2017) Let $(Q, S, \mathcal{C}, \tau)$ be a conjunctive competence model, and consider the condition requiring that for all $C_{1}, C_{2} \in \mathcal{C}$

$$
\bigcup\left\{\tau(q): \tau(q) \subseteq C_{1}, q \in Q\right\}=\bigcup\left\{\tau(q): \tau(q) \subseteq C_{2}, q \in Q\right\} \text { implies } C_{1}=C_{2} .
$$

Then the problem function $p: \mathcal{C} \rightarrow 2^{Q}$ associated to $\tau$ is injective if and only if (IC) holds true.

The so-called identifiability condition (IC) characterizes identifiability through a property of the skill map $\tau$, without referring to the associated problem function. But it is easily seen that this characterization is directly related to the latter being injective, because for $C \in \mathcal{C}$ we 
have $\tau(q) \subseteq C$ for some $q \in Q$ if and only if $q \in p(C)$. This means that the premise of (IC) holds for $C_{1}, C_{2} \in \mathcal{C}$ if and only if $p\left(C_{1}\right)=p\left(C_{2}\right)$. So, it is immediate that (IC) is both sufficient and necessary for identifiability. However, testing (IC) does not seem to provide an advantage over directly testing injectivity of $p$ when it comes to computational efficiency. Nevertheless, the following corollary to Proposition 4 provides an obvious test of the completeness of the $Q$-matrix. It is based on the contrapositive of (IC) and ensures that for any two permissible attribute patterns there is an item the response to which allows for distinguishing between them.

Corollary \%. Let $\mathbf{M}$ be the matrix of permissible attribute patterns. Then, under a conjunctive rule, the $Q$-matrix $\mathbf{Q}$ is complete if and only if for all distinct columns $\mathbf{m}_{k}, \mathbf{m}_{l}$ of $\mathbf{M}$ there is a column $\mathbf{q}_{j}$ of $\mathbf{Q}$ such that

$$
\mathbf{q}_{j} \sqsubseteq \mathbf{m}_{k}, \mathbf{q}_{j} \nsubseteq \mathbf{m}_{l}, \quad \text { or } \quad \mathbf{q}_{j} \nsubseteq \mathbf{m}_{k}, \mathbf{q}_{j} \sqsubseteq \mathbf{m}_{l} .
$$

\section{Conclusions}

In CDM, $Q$-matrix design intends to provide guidance for selecting items that allow for unique assessment of the underlying pattern of attributes from observing the item responses. Characterizing the $Q$-matrices that possess this identifiability property in the presence of dependencies between attributes is currently a highly active research area. The respective studies usually refer to Leighton et al. (2004), who list a number of so-called attribute hierarchies, which all assume that attributes are partially ordered. Two recent publications on the completeness of $Q$-matrices (as defined by Chiu et al., 2009) in conjunctive attribute hierarchy models by Cai et al. (2018) and Köhn and Chiu (2018) form the starting point of the presented developments. Both publications claim to deliver a condition that is both sufficient and necessary for the $Q$-matrix being complete. While the theorem of Cai et al. (2018) holds true within a restricted $Q$-matrix design, where consideration is confined to $Q$-matrices respecting the attribute dependencies, the theorem of Köhn and Chiu (2018) does not impose this constraint, and its necessity part is easily falsified by providing a counterexample.

The present paper aimed at deriving conditions that are sufficient and/or necessary for a complete $Q$-matrix, given dependent attributes. These characterizations should neither be constrained to particular types of $Q$-matrices, nor should they be limited to the class of attribute hierarchies. The original intention was to accomplish this mission by exploiting the close correspondence between CDM and KST (Heller et al., 2015), in particular by drawing upon results by Heller et al. $(2016,2017)$ obtained within the KST framework. Indeed, the above shows that characterizations of complete $Q$-matrices arise from directly translating these results into CDM terms according to the correspondences outlined in Section 3. But it turns out that KST can also take advantage from this enterprize. Looking at the identifiability of conjunctive competence models defined on an ordinal competence space from 
the perspective of $Q$-matrix design leads to the derivation of new conditions, like the starred witness condition $\left(\mathrm{W}^{*}-\mathcal{C}\right)$, and the strict weak witness condition $(\mathrm{swW}-\mathcal{C})$. Table 3 presents an overview over the established results. For each of the relevant corollaries the table lists the KST condition corresponding to the given criterion, it specifies the kind of structure to which the criterion applies (attribute hierarchies, or general attribute structures as introduced in Section 5), and it characterizes the status of the criterion (sufficient and/or necessary).

TABLE 3.

Overview of results on the completeness of the $Q$-matrix. The table indicates the KST condition corresponding to the given criterion, the kind of structure to which it applies, and its status.

\begin{tabular}{cccc}
\hline Result & KST Condition & Attribute Structure & Status \\
\hline Corollary 3 & $(\mathrm{~W}-\mathcal{C})$ & general & sufficient \\
Corollary 4 & $\left(\mathrm{~W}^{*}-\mathcal{C}\right)$ & hierarchy & sufficient \& necessary \\
Corollary 5 & $(\mathrm{wW}-\mathcal{C})$ & general & necessary \\
Corollary 6 & $(\mathrm{swW}-\mathcal{C})$ & hierarchy & sufficient \& necessary \\
Corollary 7 & $(\mathrm{IC})$ & general & sufficient \& necessary \\
\hline
\end{tabular}

For the attribute hierarchies considered by Leighton et al. (2004), Corollaries 4 and 6 both offer conditions that are sufficient and necessary for completeness of the $Q$-matrix. These results provide a full characterization of the class of complete $Q$-matrices for partially ordered attributes, and thereby generalize the theorem of Cai et al. (2018) beyond the limitations imposed by the restricted $Q$-matrix design. They also formulate corrected versions of the false theorem of Köhn and Chiu (2018). For general attribute structures, as they are captured by arbitrary matrices of permissible attribute patterns (containing the 0 -vector and 1 -vector), Corollaries 3, 5, and 7 characterize complete $Q$-matrices through sufficient, necessary, and both sufficient and necessary conditions, respectively. Corollary 3 translates the witness condition (W-C) of Heller et al. (2015, 2016) into CDM terminology. This is exactly the criterion that Cai et al. (2018) and Köhn and Chiu (2018) identified as being sufficient for completeness of the $Q$-matrix in case of attribute hierarchies. Corollary 3 makes clear that this holds for general attribute structures, too. Corollary 7 finally formulates a condition which is both sufficient and necessary for a $Q$-matrix to be complete in general attribute structures.

The results of the present paper do also contribute to the debate on a restricted vs. an independent (or, unstructured) $Q$-matrix design. First of all, they suggest to give up on the fixation on the $Q$-matrix as the entity containing all the relevant information. In case of dependencies between attributes, the matrix of permissible attribute patterns $\mathbf{M}$ has to be considered in conjunction with the $Q$-matrix $\mathbf{Q}$. It was shown that the matrix $\mathbf{M}$ can be understood as a representation of highly general attributes structures, including attribute hierarchies, but going far beyond them (cf. Section 5). Within this generalized framework it is 
argued that, from a conceptual point of view, the mutual relations between attributes are to be considered in their own right (through $\mathbf{M}$ ), and should not be linked to any particular items (by means of a $Q$-matrix). Corollary 2 frees the $Q$-matrix from the need to respect the attribute dependencies, and thus takes a clear stance for the independent $Q$-matrix design. Characterizing an item through $\mathbf{Q}$ should only refer to the attributes that are required to solve it, while their prerequisites are properly handled by predicting expected response patterns on the basis of $\mathbf{M}$. Thus, in the spirit of Leighton et al. (2004, p. 213) we might say that the $Q$-matrix together with the matrix of permissible attribute patterns represent "the attribute blueprint or cognitive specifications for test construction".

The presented results set the stage for further research. It is desirable to transfer the above mentioned characterizations of identifiability from conjunctive to disjunctive competence models by exploiting their duality. Following the same line of argumentation as above may lead to conditions warranting unique assessment of attribute patterns in general attribute structure models under a disjunctive rule. Moreover, Heller et al. (2015) pointed out that additional aspects have to be taken into account when it comes to the identifiability of probabilistic models defined upon competence models. In particular, this was showcased for the CBLIM, which corresponds to the MS-DINA (de la Torre \& Douglas, 2008), and includes equivalents of the DINA (E. H. Haertel, 1984, 1989, 1990) as well as the DINO (Templin \& Henson, 2006) as submodels. Elaborating on these results in view of the KST-CDM correspondences is a task still pending, and amounts to properly generalizing available theorems for the independent attributes case by (Gu \& Xu, 2018; Xu, 2017; Xu \& Zhang, 2016). Synergy effects from tackling this task are expected in very much the same way as they emerged in the present paper.

\section{References}

Birkhoff, G. (1937). Rings of sets. Duke Mathematical Journal, 3, 443-454.

Bolt, D. (2007). The present and future of IRT-based cognitive diagnostic models (ICDMs) and related methods. Journal of Educational Measurement, 44(4), 377-383.

Cai, Y., Tu, D., \& Ding, S. (2018). Theorems and methods of a complete Q matrix with attribute hierarchies under restricted Q-matrix design. Frontiers in Psychology, 9, 1413.

Chiu, C.-Y., Douglas, J. A., \& Li, X. (2009). Cluster analysis for cognitive diagnosis: Theory and applications. Psychometrika, 74, 633-665.

Chiu, C.-Y., \& Köhn, H.-F. (2015). Consistency of cluster analysis for cognitive diagnosis: The DINO model and the DINA model revisited. Applied Psychological Measuremen, 39, 465-479.

de la Torre, J. (2009). DINA model and parameter estimation: A didactic. Journal of Educational and Behavioral Statistics, 34, 115-130.

de la Torre, J., \& Douglas, J. (2008). Model evaluation and multiple strategies in cognitive diagnosis: an analysis of fraction subtraction data. Psychometrika, 73(4), 595-624. 
de la Torre, J., Hong, Y., \& Deng, W. (2010). Factors affecting the item parameter estimation and classification accuracy of the DINA model. Journal of Educational Measurement, 47, 227-249.

DiBello, L. V., \& Stout, W. (2007). Guest editors' introduction and overwiew: IRT-based cognitive diagnostic models and related methods. Journal of Educational Measurement, $44(4), 285-291$.

Doignon, J.-P., \& Falmagne, J.-C. (1985). Spaces for the Assessment of Knowledge. International Journal of Man-Machine Studies, 23, 175-196.

Doignon, J.-P., \& Falmagne, J.-C. (1999). Knowledge Spaces. Berlin, Heidelberg, and New York: Springer-Verlag.

Düntsch, I., \& Gediga, G. (1995). Skills and knowledge structures. British Journal of Mathematical and Statistical Psychology, 48, 9-27.

Falmagne, J.-C. (1989). A latent trait theory via a stochastic learning theory for a knowledge space. Psychometrika.

Falmagne, J.-C., \& Doignon, J.-P. (2011). Learning spaces: Interdisciplinary applied mathematics. Berlin, Heidelberg: Springer.

Gediga, G., \& Düntsch, I. (2002). Skill set analysis in knowledge structures. British Journal of Mathematical and Statistical Psychology, 55, 361-384.

Gu, Y., \& Xu, G. (2018). The sufficient and necessary condition for the identifiability and estimability of the DINA model. Psychometrika. doi: 10.1007/s11336-018-9619-8

Haertel, E., \& Wiley, D. (1994). Representation of ability structure: Implications for testing. In N. Fredriksen, R. Mislevy, \& I. Bejar (Eds.), Testing theory for a new generation of tests (p. 359-384). Hillsdale, NJ: Erlbaum.

Haertel, E. H. (1984). An application of latent class models to assessment data. Applied Psychological Measurement, 8, 333-346.

Haertel, E. H. (1989). Using restricted latent class models to map skill structure of achievement items. Journal of Educational Measurement, 26, 301-321.

Haertel, E. H. (1990). Continuous and discrete latent structure models of item response data. Psychometrika, 55, 477-494.

Heller, J., Anselmi, P., Stefanutti, L., \& Robusto, E. (2017). A necessary and suffcient condition for unique skill assessment. Journal of Mathematical Psychology, 79, 23-28.

Heller, J., \& Repitsch, C. (2008). Distributed skill functions and the meshing of knowledge structures. Journal of Mathematical Psychology, 52, 147-157.

Heller, J., Stefanutti, L., Anselmi, P., \& Robusto, E. (2015). On the link between cognitive diagnostic models and knowledge space theory. Psychometrika, 80, 995-1019.

Heller, J., Stefanutti, L., Anselmi, P., \& Robusto, E. (2016). Erratum to: On the link between cognitive diagnostic models and knowledge space theory. Psychometrika, 81, 250-251.

Heller, J., Ünlü, A., \& Albert, D. (2013). Skills, competencies and knowledge structures. In J.-C. Falmagne, C. Doble, D. Albert, D. Eppstein, \& X. Hu (Eds.), Knowledge spaces: 
Applications in education. Interdisciplinary applied mathematics. (p. 229-242). Berlin, Heidelberg: Springer.

Junker, B. W., \& Sijtsma, K. (2001). Cognitive assessment models with few assumptions, and connections with nonparametric item response theory. Applied Psychological Measurement, 25, 258-272.

Köhn, H.-F., \& Chiu, C.-Y. (2017). A procedure for assessing completeness of the Q-matrix of cognitive diagnosis models. Psychometrika, 82, 112-132.

Köhn, H.-F., \& Chiu, C.-Y. (2018). Attribute hierarchy models in cognitive diagnosis: identifiability of the latent attribute space and conditions for completeness of the Q-matrix. Journal of Classification.

Korossy, K. (1997). Extending the theory of knowledge spaces: A competence-performance approach. Zeitschrift für Psychologie, 205, 53-82.

Korossy, K. (1999). Modeling knowledge as competence and performance. In D. Albert \& J. Lukas (Eds.), Knowledge spaces: Theories, empirical research, applications (p. 103-132). Mahwah, NJ: Lawrence Erlbaum Associates.

Leighton, J. P., Gierl, M. J., \& Hunka, S. M. (2004). The attribute hierarchy method for cognitive assessment: A variation on Tatsuoka's rule-space approach. Journal of Educational Measurement, 41, 205-237.

Liu, R., Huggins-Manley, A., \& Bradshaw, L. (2017). The impact of Q-matrix designs on diagnostic classification accuracy in the presence of attribute hierarchies. Educational and Psychological Measurement, 77, 220-240.

Roussos, L. A., Templin, J. L., \& Henson, R. A. (2007). Skills diagnosis using IRT-based latent class models. Journal of Educational Measurement, 44, 293-311.

Rupp, A. A., Templin, J. L., \& Henson, R. A. (2010). Diagnostic measurement: Theory, methods, and applications. New York: Guilford Press.

Stefanutti, L., Heller, J., Anselmi, P., \& Robusto, E. (2012). Assessing local identifiability of probabilistic knowledge structures. Behavior Research Methods, 44, 1197-1211.

Tatsuoka, C. (2002). Data-analytic methods for latent partially ordered classification models. Journal of the Royal Statistical Society Series C (Applied Statistics), 51, 337-350.

Tatsuoka, C. (2009). Diagnostic models as partially ordered sets. Measurement, 7, 49-53.

Tatsuoka, K. K. (1990). Toward an integration of item-response theory and cognitive error diagnosis. In N. Frederiksen, R. Glaser, A. Lesgold, \& M. Safto (Eds.), Monitoring skills and knowledge acquisition (p. 453-488). Hillsdale: Lawrence Erlbaum Associates.

Tatsuoka, K. K., \& Boodoo, G. M. (2000). Subgroup differences on the GRE quantitative test based on the underlying cognitive processes and knowledge. In A. E. Kelly \& R. Lesh (Eds.), Handbook of research design in mathematics and science education (p. 821-857). Mahwah, NJ: Erlbaum.

Templin, J. L., \& Bradshaw, L. (2014). Hierarchical diagnostic classification models: A family of models for estimating and testing attribute hierarchies. Psychometrika, 79, 317-339. 
Templin, J. L., \& Henson, R. A. (2006). Measurement of psychological disorders using cognitive diagnosis models. Psychological Methods, 11, 287-305.

Xu, G. (2017). Identifiability of restricted latent class models with binary responses. The Annals of Statistics, 45, 675-707.

Xu, G., \& Zhang, S. (2016). Identifiability of diagnostic classification models. Psychometrika, 81, 625-649. 


\section{Appendix}

Considering the DINA model, Köhn and Chiu (2018, Theorem 1) base their derivations on a somewhat different notion of completeness introduced by Köhn and Chiu (2017). It refers to the expectation of the response patterns, and thus is not independent of the probabilistic assumptions of the DINA model. The equivalence of these notions was established for independent attributes only (Chiu \& Köhn, 2015, Lemma 1). The following proves it for any general attribute structure. In order to be able to draw upon KST results, the proof is formulated within the KST framework. This means that we consider a conjunctive CBLIM, which was shown to be equivalent to the DINA model defined on an arbitrary conjunctive competence model $(Q, S, \mathcal{C}, \tau)$ (Heller et al., 2015). To prepare the result, some notions are to be introduced.

The Basic Local Independence Model (BLIM; Falmagne, 1989; Doignon \& Falmagne, 1999) specifies a (marginal) distribution $P_{\mathcal{K}}$ on the states of a given knowledge structure $\mathcal{K}$, by which the marginal distribution $P_{\mathcal{R}}$ on the set of observed response patterns $\mathcal{R}=2^{Q}$ is predicted by

$$
P_{\mathcal{R}}(R)=\sum_{K \in \mathcal{K}} P(R \mid K) \cdot P_{\mathcal{K}}(K) .
$$

for all $R \in \mathcal{R}$. Here, the conditional probabilities $P(R \mid K)$ satisfy the following condition: For each $q \in Q$ there are real constants $0 \leq \beta_{q}<1$ and $0 \leq \eta_{q}<1$ such that for all $R \in \mathcal{R}$ and $K \in \mathcal{K}$

$$
P(R \mid K)=\left(\prod_{q \in K \backslash R} \beta_{q}\right) \cdot\left(\prod_{q \in K \cap R}\left(1-\beta_{q}\right)\right) \cdot\left(\prod_{q \in R \backslash K} \eta_{q}\right) \cdot\left(\prod_{q \in Q \backslash(R \cup K)}\left(1-\eta_{q}\right)\right) .
$$

The constants $\beta_{q}$ and $\eta_{q}$ are interpreted as the probabilities of a careless error and a lucky guess, respectively, on item $q$. The correspondence to the slipping and guessing parameters of the DINA model and to its assumption of local stochastic independence is obvious (see Heller et al., 2015, for details).

Skills are easily integrated into this probabilistic framework. Given a conjunctive competence model $(Q, S, \mathcal{C}, \tau)$, consider a probability distribution $\pi_{\mathcal{C}}$ on the states of competence structure $\mathcal{C}$. With $p$ the problem function associated to the skill map $\tau$, let $p^{-1}(\{K\})$ denote the preimage of the subset $\{K\}$ of $\mathcal{K}$ under $p$. Then

$$
P_{\mathcal{K}}(K)=\sum_{C \in p^{-1}(\{K\})} P_{\mathcal{C}}(C)
$$

defines a probability distribution on the knowledge states of $\mathcal{K}$, on which a BLIM may be built as outlined above. This means that a Competence-Based Local Independence Model (CBLIM) satisfies the equations

$$
P_{\mathcal{R}}(R)=\sum_{C \in \mathcal{C}} P(R \mid C) \cdot P_{\mathcal{C}}(C)
$$


for all $R \in \mathcal{R}$, and

$$
P(R \mid C)=P(R \mid p(C))
$$

for all $C \in \mathcal{C}$, where the right hand side of the latter equation is given by (14). The BLIM defined on the delineated knowledge structure $\mathcal{K}=p(\mathcal{C})$ will be called the BLIM induced by the CBLIM. If the competence model $(Q, S, \mathcal{C}, \tau)$ is assumed to be conjunctive then the CBLIM is said to be conjunctive, too.

Given a conjunctive CBLIM defined on the conjunctive competence model $(Q, S, \mathcal{C}, \tau)$ the definition used by Köhn and Chiu (2018) amounts to

$$
\left(P\left(R \mid C_{1}\right)=P\left(R \mid C_{2}\right) \text { for all } R \in \mathcal{R}\right) \text { implies } C_{1}=C_{2}
$$

for all competence states $C_{1}, C_{2} \in \mathcal{C}$. Due to (16) we need to consider the stochastic matrix $P(R \mid K)$ for all $R \in \mathcal{R}$ and all states $K$ in the delineated knowledge structure $\mathcal{K}$. The proof of Theorem 1 in Stefanutti, Heller, Anselmi, and Robusto (2012) shows that for the BLIM (and so, in particular, for the DINA model) this matrix has full rank. Thus, for $K_{1}, K_{2} \in \mathcal{K}$, whenever $P\left(R \mid K_{1}\right)=P\left(R \mid K_{2}\right)$ holds for all $R \in \mathcal{R}$, then $K_{1}=K_{2}$. Given $p\left(C_{1}\right)=K_{1}$ and $p\left(C_{2}\right)=K_{2}$ for $C_{1}, C_{2} \in \mathcal{C}$ this implies $C_{1}=C_{2}$ if the problem function $p$ associated to $\tau$ is injective. So, for the DINA model the definition of completeness by Köhn and Chiu (2017) reduces to that of Chiu et al. (2009). 